\title{
The determination of material parameters from superplastic inflation tests
}

\author{
Jung-Ho Cheng \\ Department of Mechanical Engineering, National Taiwan University, Taipei, Taiwan 10617, ROC
}

Received 17 December 1994

\begin{abstract}
Industrial summary
A procedure for determining parameters in a selected material model from a superplastic blow-forming test is presented. Emphasis is placed on the relative ease of the procedure and the lower cost of the test equipment required compared to the more involved strain-rate-controlled tensile tests. Analytical solutions are derived to correlate the true stresses and true strain rates from the experimental measurements of the inflation height of a hemispherical dome. Several material models are chosen to fit the stress-strain rate data obtained from the proposed procedures. The material models, together with the associated parameters, are implemented as user's sub-routines for the commmercial finite-element program, ABAQUS. Verification of the proposed approach is carried out by comparing the numerical solutions obtained from the program with the experimental results.
\end{abstract}

Keywords: Material-testing systems; Superplastic forming; Hemispherical inflation; Constitutive models; Power laws; Strain-rate sensitivity; Finite-element simulation

\section{Introduction}

Superplasticity is usually characterized by the high strain-rate sensitivity of the flow stress, $m$, and its variation with strain rate, temperature and grain size [1-4]. Conventionally, most of the stress-strain and stress-strain data presented in the literature were obtained from constant cross-head speed tensile testing and measuring the steady-state load. This approach does not, however, provide proper stress-strain rate data for large-deformation analysis, since during constant speed testing, if all other effects remain unchanged, the true strain rate decreases as the specimen elongates and, thus, the flow stress would decrease with increasing strain. The error can be severe, especially in a superplastic forming process as elongations of hundreds of percent are common. It is, therefore, possible for the strain rate to decrease by more than an order of magnitude during constant speed testing.

Ideally, if the microstructure of the material remains constant, the true flow stress can be obtained by carrying out constant strain rate tensile tests for a range of strain rates, measuring the steady-state load. Since the true strain can be defined in terms of the current length of the specimen, $l$, as $\varepsilon=\ln \frac{l}{l_{0}}$,

a constant strain rate tensile test can be realized by controlling the cross-head speed to give

$l=l_{0} \dot{\varepsilon} \exp (\dot{\varepsilon} t)$

where $l_{0}$ is the initial length, $\dot{\varepsilon}$ is the desired strain rate and $t$ represents time.

The above procedure is, nevertheless, not appropriate for obtaining superplastic material parameters, since most engineering materials are microstructurally unstable at elevated temperatures where superplastic forming processes take place. The flow stress often increases with increasing strain due to the effects of grain growth. On the other hand, the flow stress may decrease with strain, particularly at high strains, because of the development of cavitation damage. It was in this respect that step strain rate or strain rate jump tests were developed to determine the stress-strain rate relations of a constant structure [5].

To measure $m$ over a range of strain rates, a tensile specimen is deformed at a particular velocity (or strain rate) until a steady state is attained. The cross-head speed is then reduced to a low value and the load is measured after being allowed to stabilize. Repeated 
incremental increases in cross-head speed allow the load to be measured over a range of strain rates [6]. Several procedures have been proposed for determining the relevant true stresses and strain rates at each cross-head speed and, hence, provide the desired true stress-strain rate relationship [7-14].

All the procedures discussed so far are based on uniaxial tension tests. In order to obtain reliable data for superplastic materials, the experimental set-ups for performing the tests are usually costly, time consuming and error prone. Some important considerations in a typical uniaxial tensile test are

(1) High temperatures should be maintained uniformly throughout the gauge length of the test specimen.

(2) A protective gas, such as argon gas, should be applied to the test chamber to avoid oxidation of the surface of the specimen. The test chamber should first be evacuated and then sealed well in order not to consume excessive amount of argon gas. Sealing can be difficult at high temperatures, especially between the pull rods and the chamber wall.

(3) Compounding the above difficult sealing problem are the alignment of the pull rods and the accurate control of the cross-head speed of the machine for a wide range of very low strain rates.

The test equipment that meets the above requirements usually costs in the region of US\$200 000. Although it is not an enormous amount, not every laboratory where the tests are needed can afford such an investment. It is, thus, desirable to have a procedure which costs less yet still provides reliable stress-strain rate data.

In this paper, the superplastic inflation of a flat sheet into a hemispherical dome is adopted to perform biaxial tensile tests. Analytical solutions of the inflated sheet at equilibrium are derived to correlate the true stresses and true strain rates from the experimental results. The data are fitted with several models for comparison. The choice of a particular model should be based not only on the best fit of the data, but also on the best representative of the derivatives of stress with respect to the strain rate, since these are the values needed for the Newton method that is commonly employed for solving non-linear equations.

The acquired material models and the associated constants are implemented in a commercial finite-element program, ABAQUS, via its user sub-routine capability. Numerical results obtained from the program are compared with the experimental results.

\section{Analytical derivations of stress-strain rate relationships}

Fig. 1 presents a schematic diagram of gas pressure forming in a cylindrical die with one end closed. A thin circular sheet is clamped by a hydraulic press and pressurised argon gas is applied to the sheet to form it into a hemispherical dome.

In order to obtain the stress-strain rate relationship from an experiment, there is the need to be able to measure true stress versus true strain rate at any point in time. This is not simple to do for the test shown in Fig. 1. When the sheet deforms in a closed chamber, what can be accurately measured and recorded as a function of time is usually the height of the dome using an LVDT device. However, using geometric relationships and the relationship between the pressure and the hoop stress, the necessary data can be derived from the measured heights.

Assuming that there is no variation of stress through the thickness of a thin-walled pressure vessel, the hoop stress $\sigma_{1}$ can be related to the applied pressure $p$ by

$\sigma_{1}=\frac{p \rho}{2 s}$,

where $\rho$ is the radius of curvature and $s$ is the thickness of the sheet.

If the von Mises' effective stress criterion

$\bar{\sigma}=\frac{1}{\sqrt{2}}\left[\left(\sigma_{1}-\sigma_{2}\right)^{2}+\left(\sigma_{2}-\sigma_{3}\right)^{2}+\left(\sigma_{3}-\sigma_{1}\right)^{2}\right]^{1 / 2}$,

is used, where $\sigma_{2}$ is the meridional stress and $\sigma_{3}$ is the thickness stress, the above relationship can be expressed as

$\bar{\sigma}=\frac{p \rho}{2 s}$,

since, from symmetry, $\sigma_{2}=\sigma_{1}$, and $\sigma_{3} \approx 0$ for a thin membrane.

If the principal stresses and strains are related by Nadai's equation [15] by

$\varepsilon_{1}=\frac{1}{E_{\mathrm{p}}}\left[\sigma_{1}-\frac{\sigma_{2}+\sigma_{3}}{2}\right]$

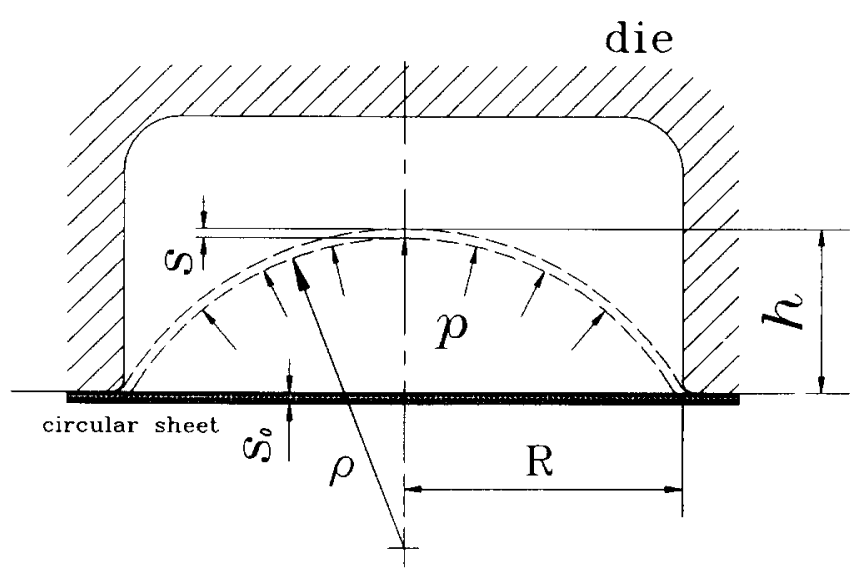

Fig. 1. Schematic diagram of a superplastic free-inflation process. 
$\varepsilon_{2}=\frac{1}{E_{\mathrm{p}}}\left[\sigma_{2}-\frac{\sigma_{1}+\sigma_{3}}{2}\right]$

$\varepsilon_{3}=\frac{1}{E_{\mathrm{p}}}\left[\sigma_{3}-\frac{\sigma_{1}+\sigma_{2}}{2}\right]$

then from $\sigma_{1}=\sigma_{2}$, and $\sigma_{3}=0$

$\varepsilon_{1}=\varepsilon_{2}$

and, from constancy of volume, $\varepsilon_{1}+\varepsilon_{2}+\varepsilon_{3}=0$

$\varepsilon_{3}=-2 \varepsilon_{1}$

where $E_{\mathrm{p}}$ is the plastic modulus, and $\varepsilon_{1}, \varepsilon_{2}, \varepsilon_{3}$ are the hoop strain, meridional strain and thickness strain, respectively. Using the above strain relations, the von Mises' effective strain can be written as

$$
\begin{aligned}
\bar{\varepsilon} & =\frac{\sqrt{2}}{3}\left[\left(\varepsilon_{1}-\varepsilon_{2}\right)^{2}+\left(\varepsilon_{2}-\varepsilon_{3}\right)^{2}+\left(\varepsilon_{3}-\varepsilon_{1}\right)^{2}\right]^{1 / 2}, \\
& =\varepsilon_{3}
\end{aligned}
$$

Since the thickness strain is defined as

$\varepsilon_{3}=\ln \frac{s}{s_{0}}$,

the effective strain rate can be expressed as

$\overline{\dot{\varepsilon}}=\frac{\dot{s}}{S}$,

where $s_{0}$ is the initial thickness.

From the geometry shown in Fig. 1, it is found that

$\rho^{2}=R^{2}+(\rho-h)^{2}$

or

$\rho=\frac{R^{2}+h^{2}}{2 h}$

where $R$ is the radius of the die opening.

The area $A$ of the deformed membrane, of radius of curvature $\rho$, at any instant can be written as

$A=2 \pi \rho h$

If the dome is further assumed to maintain a uniform thickness throughout the inflation process, then from constancy of volume

$A s=A_{0} s_{0}$

where $A_{0}=\pi R^{2}$ and is the initial area corresponding to $A$ of the sheet.

Thus, the instantaneous thickness can be obtained from

$s=\frac{A_{0} s_{0}}{2 \pi \rho h}=\frac{R^{2} s_{0}}{R^{2}+h^{2}}$,

and the effective strain rate can then be written as

$\overline{\dot{\varepsilon}}=\frac{\dot{s}}{S}=\frac{-2 h \dot{h}}{R^{2}+h^{2}}$
Hence, if a material is chosen, e.g., $\bar{\sigma}=\phi(\bar{\varepsilon})$, then the stress-strain rate relationship can be expressed as

$\frac{p\left(R^{2}+h^{2}\right)^{2}}{4 h R^{2} s_{0}}=\phi\left(\frac{-2 h \dot{h}}{R^{2}+h^{2}}\right)$

in which $h$, as a function of time, is the only unknown. Once $h$ is acquired from the experiments, the necessary parameters in the model $\phi$ can then be determined.

The above derivations are based on the assumption of uniform thickness, Eq. (18). Since the polar region thins down at a faster rate, the thickness of the sheet will not remain uniform during the inflation process. However, from the force balance, it is evident that Eq. (21) is applicable to any point in the thin sheet and is independent of the uniformity of the thickness. The relationship in Eq. (21) is, thus, a representation of average true stress versus average true strain rate. Corrections of the non-uniform thinning will be discussed at the end of the paper.

\section{Procedure for determining material data from the inflation test}

The procedure for determining material parameters from superplastic inflation tests is illustrated in Fig. 2. Under a given constant pressure, the heights of the dome are measured by an LVDT device and recorded as a function of time. The data is curve-fitted to give a function relationship $h(t)$, and then differentiated with respect to time to obtain $\dot{h}$. Once a material model $\bar{\sigma}=\phi(\bar{\varepsilon})$ is chosen, the associated material parameters of the model can be determined from least-squares curve fitting by substituting the data sets $\left\{h\left(t_{i}\right), t_{i}\right\}_{i=1}^{n}$ and $\left\{\dot{h}\left(t_{i}\right), t_{i}\right\}_{i=1}^{n}$ into Eq. (21). In the above procedure, $t_{i}$ represents the times when measurements were taken and $n$ is the number of points selected for data fitting.

A simple program, written in Macsyma [16] language, is included in Appendix A for performing the tasks shown in Fig. 2. In the program, the stress and strain rates are computed only for the earlier stages of the experimental data. This has significantly improved the accuracy of the derived stress-strain rate data, since the thickness of the dome remains relatively uniform at low values of $h$.

The pressure for the first experiment is set arbitrarily at a conservative value. Sometimes this may require a trial process because, if the pressure level is too high, the strain rate may exceed the superplastic limit, which can lead to blowout of the dome. After the first experiment, other pressure values may also be applied, which can be estimated from Eq. (21), with the required material parameters, so as to ensure that the desired strain rate range is covered.

Although the material is not a constant structure, if the stress--strain relationship is determined in this way, 


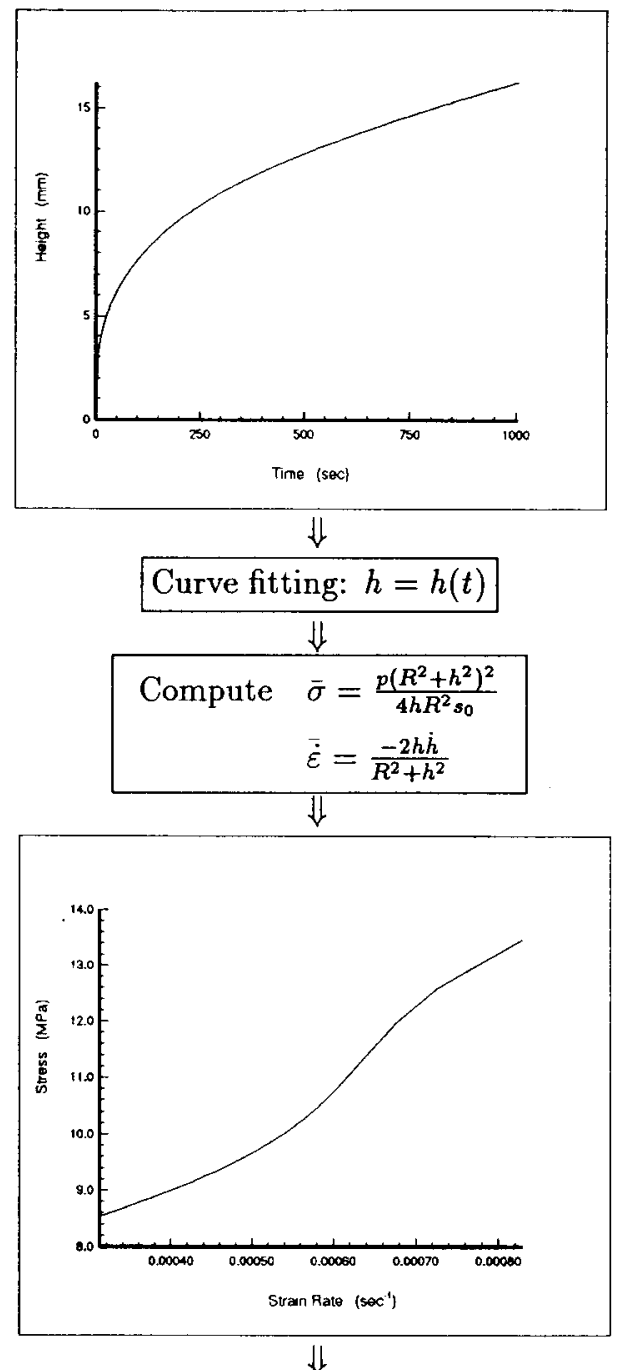

Choose a stress-strain rate model: e.g.

$\log \bar{\sigma}=\log K+m \log \overline{\dot{\varepsilon}} \quad\left(\bar{\sigma}=K \bar{\varepsilon}^{m}\right)$ or $\log \bar{\sigma}=\log B_{0}+B_{1} \log \bar{\varepsilon}+B_{2} \log \overline{\dot{\varepsilon}}^{2}+B_{3} \log \bar{\varepsilon}^{3}+\ldots$

$\begin{array}{ll}\Downarrow \\ \text { Curve fitting } \Rightarrow \quad \mathrm{K} \& \mathrm{~m} \text { or } \\ & B_{0}, B_{1}, B_{2}, B_{3}, \ldots\end{array}$

Fig. 2. The procedure for determining material parameters from superplastic inflation tests.

the discrepancy between the experimental and numerical predictions, using the parameters obtained from this procedure, is generally insignificant, as will be demonstrated later.

\section{Experimental set-ups}

The device for performing a superplastic inflation test is illustrated schematically in Fig. 3. The basic components of the system include
(1) A furnace chamber provided with a proper upper temperature limit (in these experiments, a capacity of $1000{ }^{\circ} \mathrm{C}$ or beyond is required).

(2) Upper and lower dies enclosed in the chamber, the inlet gas and vent systems of which are shown in Fig. 4. Cooling water is introduced as shown to prevent the plate and LVDT from overheating. The cross-section of the upper die is also depicted in Fig. 4. A tube is welded to the center of the die, which is reserved for installing the LVDT device. Thermocouples are embedded in both dies through the path as shown in the figure. Thus, the temperatures of the dies instead of the workpiece are measured. Calibration has been made to obtain the temperature of the workpiece from the measured data.

(3) An LVDT is used for measuring the displacement, and is attached to an extension rod which goes through the upper die and touches the top surface of the sheet. The gap between the wall of the die and the extension rod is sealed with an $O$ ring to prevent leaking of the protective argon gas from the upper die.

(4) An oil pressure system to press the two dies together.

(5) A vacuum system, installed for purging the air before the argon gas is applied. This may not be necessary, but is helpful in reducing the amount of argon gas used.

The cost of such a system is around US $\$ 15000$, less than one-tenth of the value of the more sophisticated and high precision controlled-tensile-test systems. In the following, the properties of $\mathrm{Ti}-6 \mathrm{Al}-4 \mathrm{~V}$ are discussed, although other materials, such as $\mathrm{Ti}-$ 6Al-6V-2Sn, Al7475, and Al8289, have been investigated.

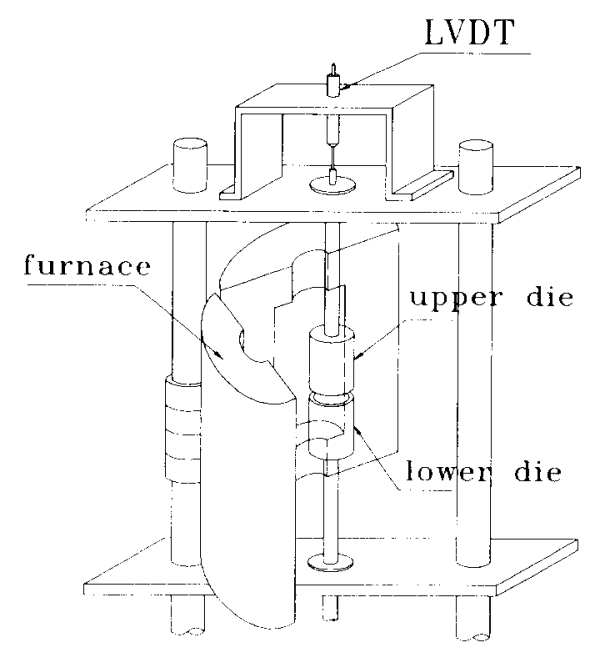

Fig. 3. Schematic representation of the experimental set-up. 

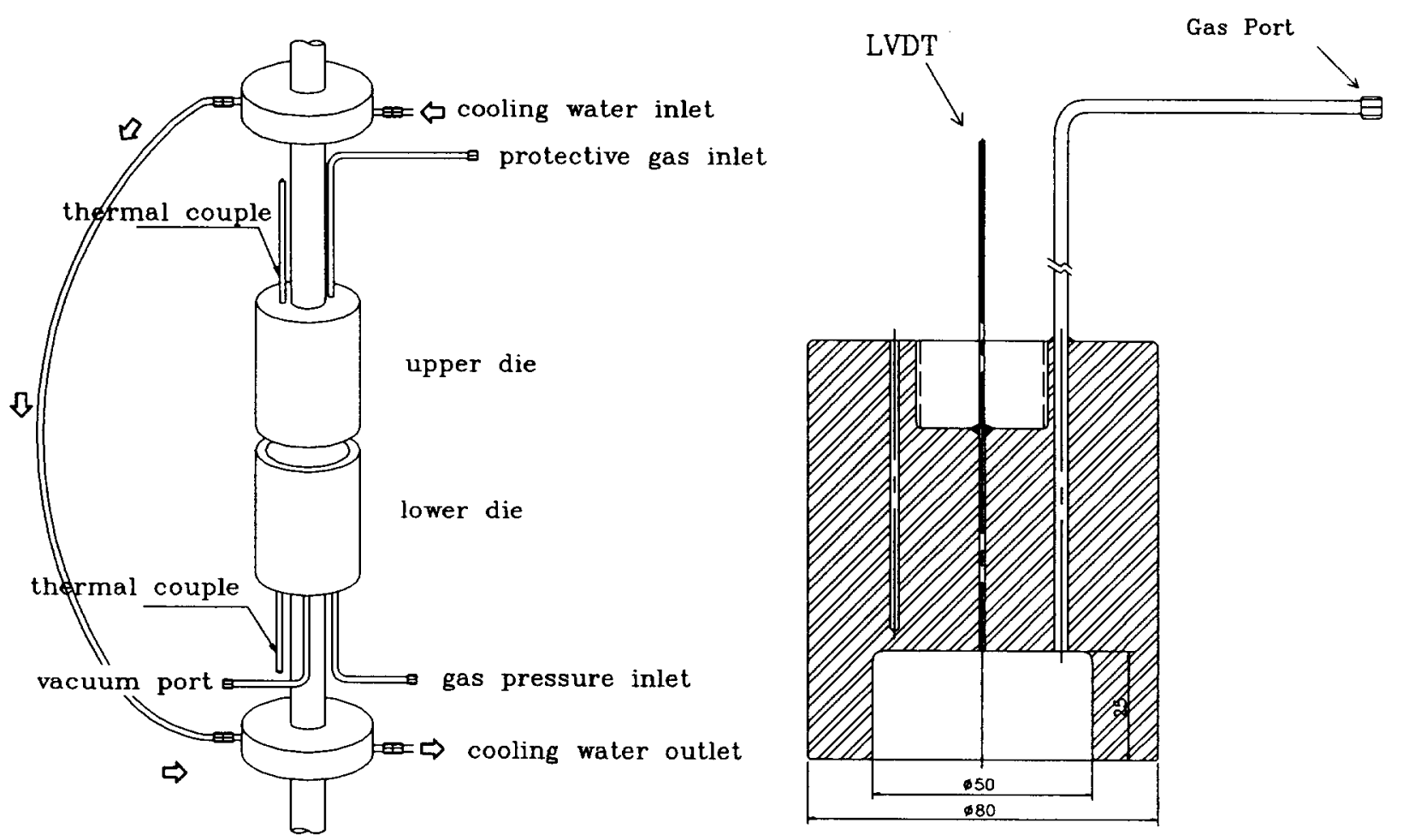

Fig. 4. (a) Detailed layouts of the upper and lower dies, cooling water and pressure paths and (b) the cross-section of the upper die.

\section{Experimental results}

As shown in Fig. 5, a circular Ti-6Al-4V sheet of $1 \mathrm{~mm}$ thickness and $70 \mathrm{~mm}$ diameter, of which the $10 \mathrm{~mm}$ wide outer rim is compressed between the two dies, is subjected to argon gas at $60 \mathrm{psi}$ from underneath. The radius of the die opening is $50 \mathrm{~mm}$. In order to prevent the sheet from sinking into the lower die due to its own weight, before the pressure is applied, a cylindrical block with a diameter smaller than the die opening is placed in the lower die. The grain size of the sheet is under $10 \mu \mathrm{m}$. The experiments are carried out at $925^{\circ} \mathrm{C}$.

During the experiment, the change in height of the sheet is measured from LVDT and recorded as a func-
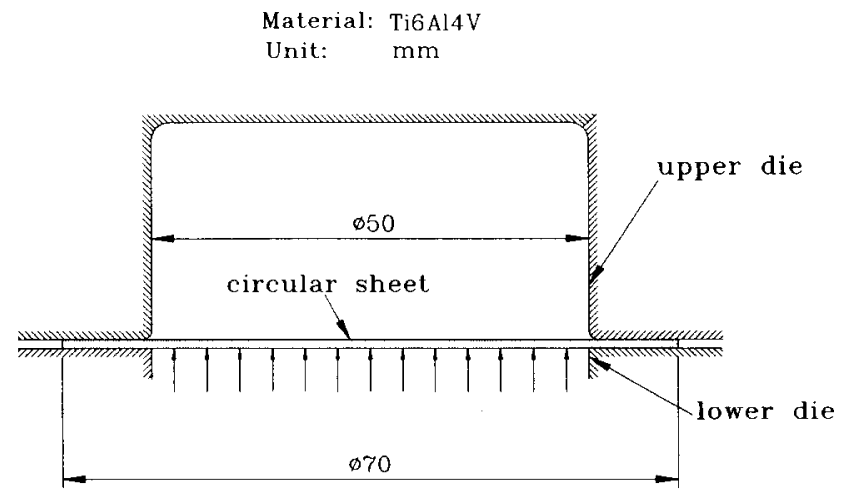

Fig. 5. Dimensions of the specimens tor the superplastic free-bulging experiment. tion of the inflation time. Fig. 6 shows typical experimental results.

Using the procedure described in the previous section, with the data given in Fig. 6, the parameters of the power law model

$\bar{\sigma}=K \overline{\bar{\varepsilon}}^{m}$,

can be found, i.e., $K=1030.78 \mathrm{MPa}$ and $m=0.61305$.

The power law represents a linear relationship between the stress and strain rate in logarithmic space, this being a popular choice in many of the analysis programs owing to its simplicity. In these programs, the parameters are treated as 'constants' or, at most, as functions of temperature and, thus, characterize a lumped-effect over a particular range of strain rates. Due to the pronounced strain-rate sensitivity of superplastic materials, the parameters $K$ and $m$ are, however, strongly dependent upon the strain rate.

To obtain $m$ as a variation of strain rate, the relationship

$m=\frac{\mathrm{d} \log \bar{\sigma}}{\mathrm{d} \log \overline{\dot{\varepsilon}}}$

can be used. Curve fitting of stress $\left(=p\left(R^{2}+h^{2}\right)^{2} /\right.$ $\left.4 h R^{2} s_{0}\right)$ versus strain rate $\left(=-2 h \dot{h} / R^{2}+h^{2}\right)$ in logarithmic space at various points of height would have to be carried out before the above differentiation could be performed. Nevertheless, it is possible to bypass the curve fitting by directly differentiating both the stress and strain rate with respect to time first, and then 


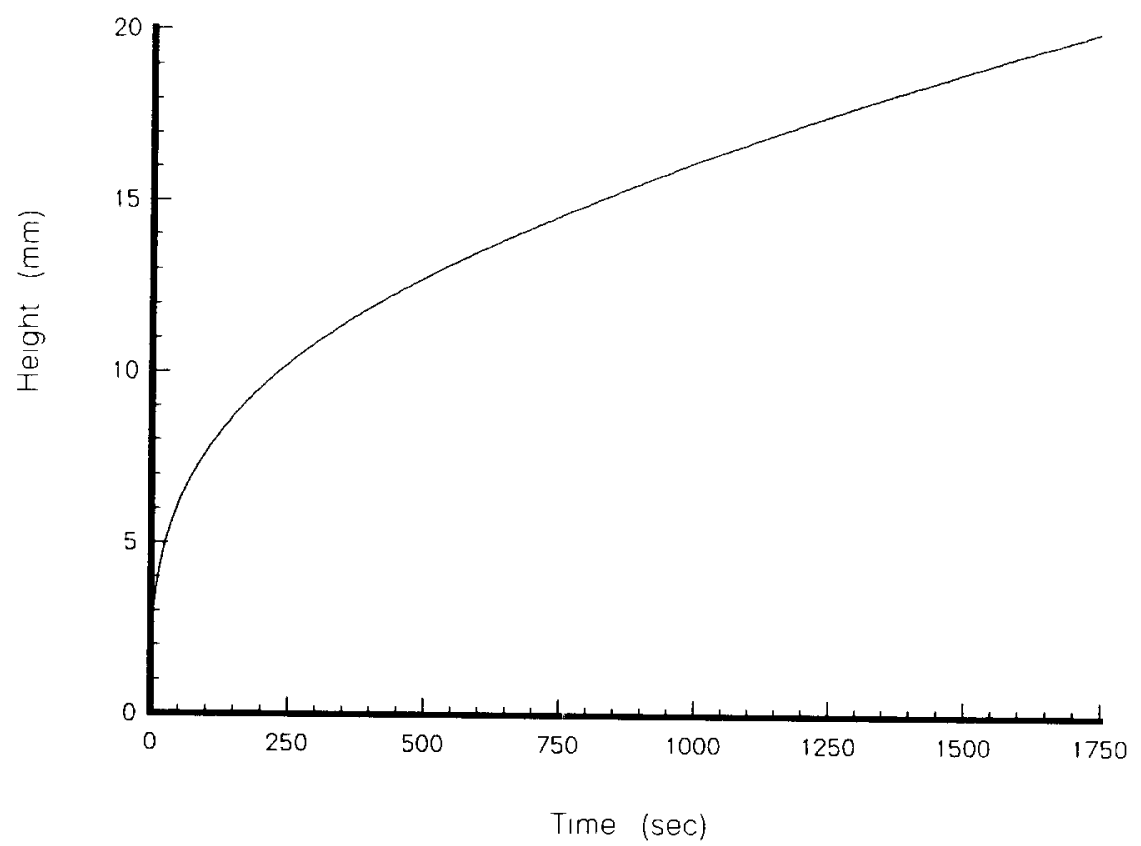

Fig. 6. Measured heights versus time from a typical experiment, the applied pressure being $60 \mathrm{psi}\left(1 \mathrm{psi}=26.89 \mathrm{kN} \mathrm{m}^{-2}\right)$.

dividing the stress derivative by the strain rate derivative, i.e.

$m=\frac{\mathrm{d} \log \bar{\sigma} / \mathrm{d} t}{\mathrm{~d} \log \bar{\varepsilon} / \mathrm{d} t}$

$=-\frac{(\mathrm{d} h / \mathrm{d} t)^{2}\left(r^{2}-3 h^{2}\right)}{\left(h\left(\mathrm{~d}^{2} h / \mathrm{d} t^{2}\right)+(\mathrm{d} h / \mathrm{d} t)^{2}\right) r^{2}+h^{3}\left(\mathrm{~d}^{2} h / \mathrm{d} t^{2}\right)-h^{2}(\mathrm{~d} h / \mathrm{d} t)^{2}}$

The presently-used version of the Macsyma program is given in Appendix B. There is no need to type in the explicit form of Eq. (25), as Macsyma can perform the differentiation in Eq. (24) symbolically to yield Eq. (25). The computed stress $\bar{\sigma}$ and $m$ as functions of strain rate $\overline{\dot{\varepsilon}}$ are shown in Fig. 7.

Newton's iteration scheme is generally used in a finite-element implementation of a non-linear solver. In such an approach not only is the relationship between stress and strain rate needed, it is also necessary to supply the derivative of stress with respect to strain rate. Adopting the power law, whilst inputing both $m$ and $K$ as functions of strain rate, is not a very convenient choice. Instead, it is more appropriate to use a higher-order polynomial form between the logarithmic stress and logarithmic strain rate, for example:

$\log \tilde{\sigma}=A_{0}+A_{1} \log \overline{\dot{\varepsilon}}+A_{2} \log \bar{\varepsilon}^{2}+A_{3} \log \bar{\varepsilon}^{3}+\ldots$,

the material parameters $A_{0}, A_{1}, A_{2}$, and $A_{3}$ being functions of temperature alone. The above relationship can be made to yield excellent agreement with experimental data over a wide span of strain rates. The strain-hardening effect can also be included by adding a term $B \bar{c}$ to the stress value, $\bar{\sigma}$.

Fig. 8 depicts the results of first-to-fourth-order fitting of the stress and strain rate relationship shown in Fig. 7. It is evident that the fourth-order polynomial gives the best representation of the stress variation with respect to strain rate.

In the ABAQUS program, when the sub-routine that describes the material creep model is called, a stress value of a particuar integration point is passed in, the sub-routine needing to return the corresponding strain rate, as well as the derivative of strain rate with respect to stress. Thus, as an alternative to Eq. (25), in the current work, the following relationship:

$\log \overline{\hat{\varepsilon}}=B_{0}+B_{1} \log \bar{\sigma}+B_{2} \log \bar{\sigma}^{2}+B_{3} \log \bar{\sigma}^{3}+B_{4} \log \bar{\sigma}^{4}$

is adopted, the material parameters $B_{0}, B_{1}, B_{2}$, and $B_{3}$ simply being constants for a specific temperature. By merely changing the second to the last line of Appendix A into 'sol:lsq $(y, x, 4)$;', the program yields

$$
\begin{aligned}
& B_{0}=-385.777, \quad B_{1}=520.665, \quad B_{2}=-259.498, \\
& B_{3}=54.3646, \quad B_{4}=-3.85909 .
\end{aligned}
$$

The positions of the arguments, $x$ (representing $\log \bar{\varepsilon}$ ) and $y$ (representing $\log \bar{\sigma}$ ), have been switched to indicate that the strain rate is now expressed as a function of stress. The number ' 4 ' in the above least-squares (Isq) curve fitting means that a fourth-order polynomial has been chosen. 


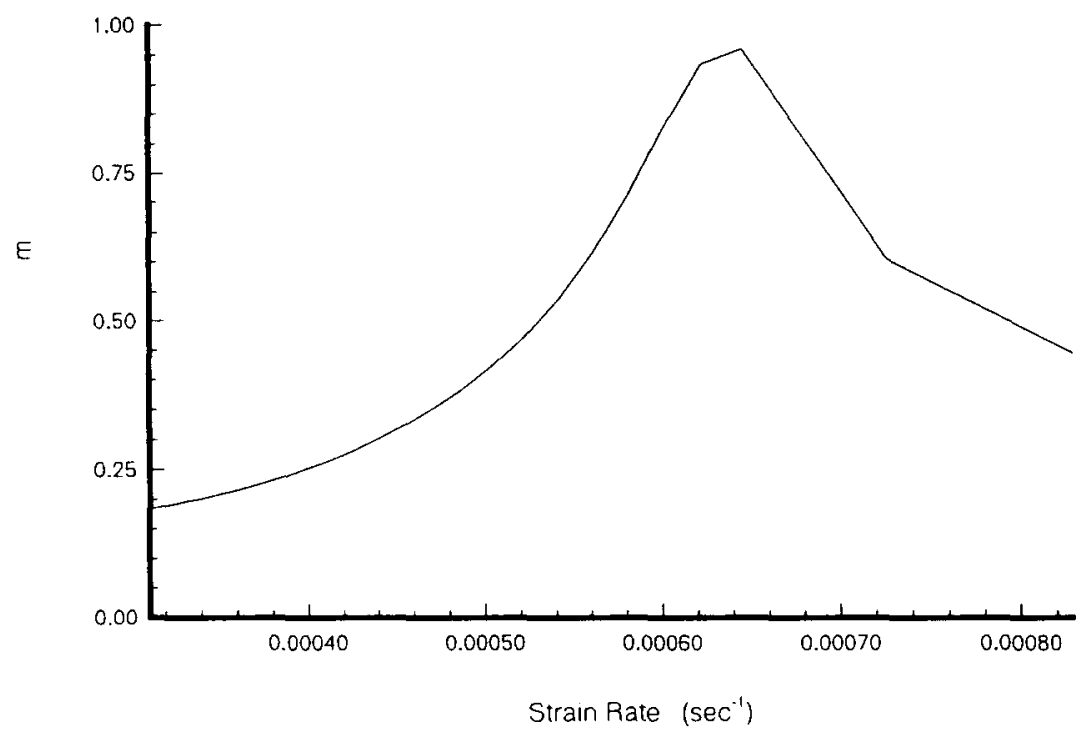

(a)

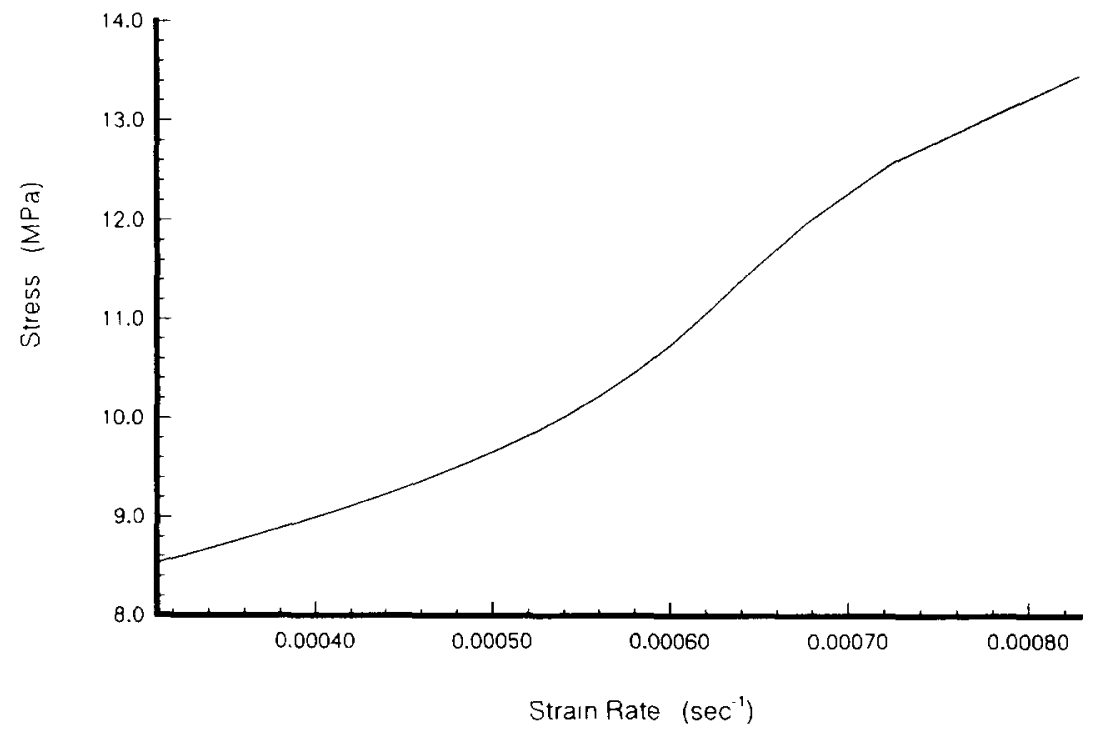

(b)

Fig. 7. (a) Computed values of $m$ versus strain rate and (b) stress versus strain rate obtained from the procedure outlined in Appendix B.

\section{Verification of proposed method}

The finite-element model used to simulate superplastic free inflation of a circular sheet into a hemispherical dome is shown in Fig. 9. The initial thickness of the blank is $1 \mathrm{~mm}$. Due to symmetry, only half of the sheet is modelled with four-noded axisymmetric elements. Both the power law, Eq. (22), and the fourth-orderpolynomial form, Eq. (27), are implemented in ABAQUS's user sub-routine CREEP [17].

Also illustrated in Fig. 9, are the deformed configurations from numerical simulation using the fourth-order model. The deformed specimen and its cut-out after
$30 \mathrm{~min}$ of free inflation is also included in the same figure for comparison.

The heights of the inflated sheet as functions of time are shown in Fig. 10, which indicates excellent agreement between the predicted and measured results, especially with the fourth-order polynomial stress-strain rate relationship. Fig. 11 compares the computed and measured thickness distributions at several locations along the arc length of the hemisphere.

Fig. 12 illustrates the results obtained from three other different constant pressures, namely, 20, 90, and 140 psi. The finite-element predictions of the inflation height using the fourth-order polynomial stress-strain rate 


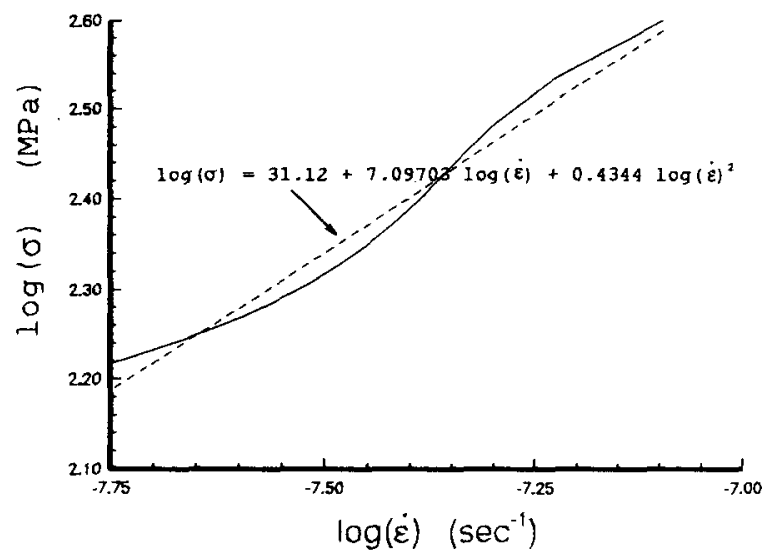

(a)

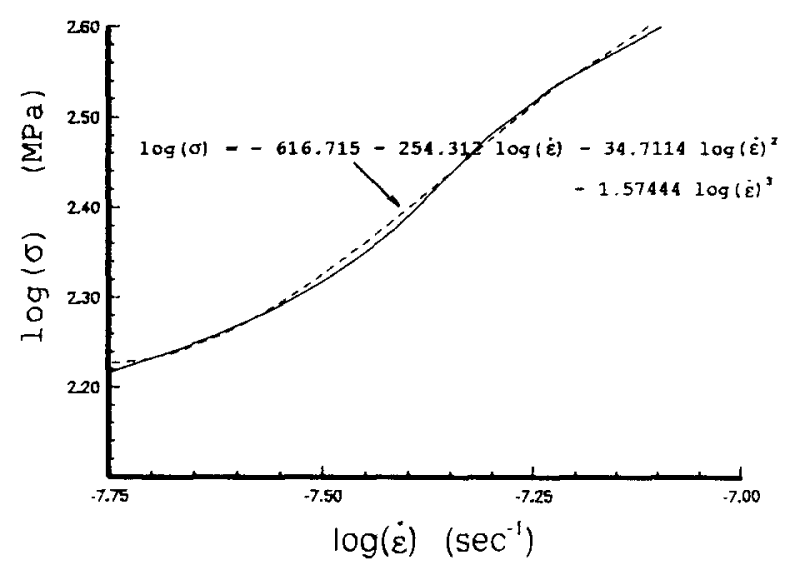

(c)

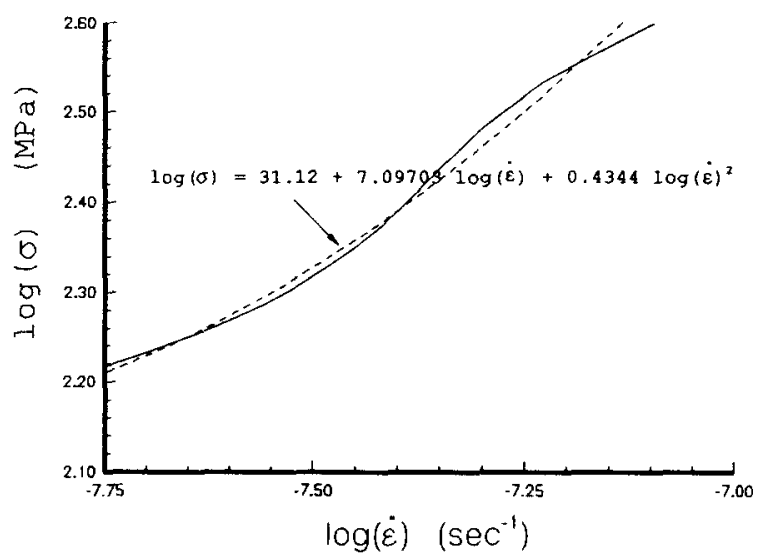

(b)

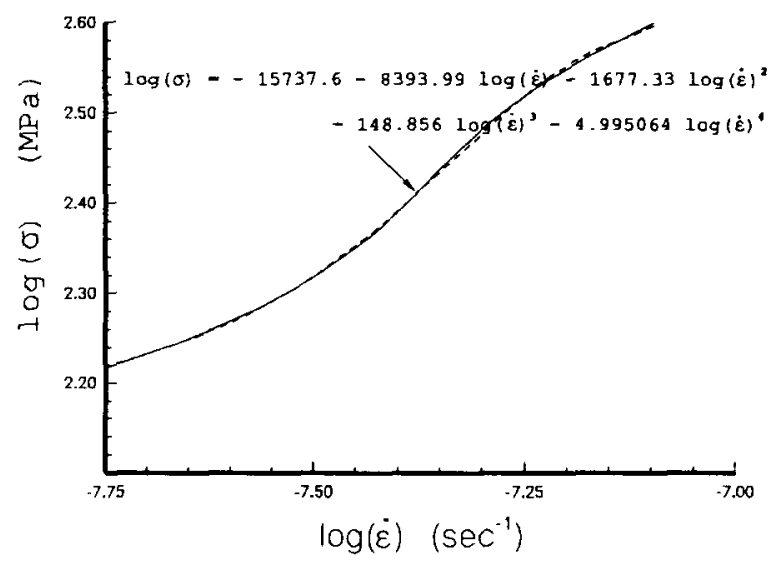

(d)

Fig. 8. Curve fitting of the stress-strain rate data shown in Fig. 7 using four different orders of polynomial.

relationship are seen to compare favorably with the measured data.

\section{Corrections for non-uniform thinning}

The above material parameters were obtained from Eq. (21), assuming uniform thinning of the bulging sheet. However, the true variation of the thickness along the arc length in the hoop direction generally follows

$s(l)=s_{\mathrm{p}}+\frac{s_{\mathrm{e}}-s_{\mathrm{p}}}{L^{2}} l^{2}$,

where subscripts $\mathrm{e}$ and $\mathrm{p}$ denote equator and pole, respectively, and $L$ is the current arc length of the deformed sheet. This parabolic relationship has been observed by Ghosh and Hamilton [18] experimentally and by Cheng [19] from finite-element results. To determine the dependence of $s_{\mathrm{e}}$ on $s_{\mathrm{p}}$, the procedure is as follows.
The relationship between the effective strain $\bar{\varepsilon}$ and the thickness strain $\varepsilon_{3}$ given in Eq. (12) is valid only for the element at the pole, where the material is free to bulge biaxially. Due to the restriction of expansion imposed by the die wall at the equator, the meridional strain at the equator is $\left(\varepsilon_{2}\right)_{e}=0$. Therefore, from constancy of volume

$\left(\varepsilon_{3}=-\varepsilon_{1}\right)_{\mathrm{e}}$

With these relationships, the von Mises' effective strains and stresses at the equator and pole can be expressed respectively as follows:

$\bar{\varepsilon}_{\mathrm{e}}=\frac{2}{\sqrt{3}}\left(\varepsilon_{3}\right)_{\mathrm{e}}$,

$\bar{\varepsilon}_{\mathrm{p}}=\left(\varepsilon_{3}\right)_{\mathrm{p}}$,

or in rate form

$\overline{\dot{\varepsilon}}_{e}=\frac{2}{\sqrt{3}}\left(\dot{\varepsilon}_{3}\right)_{\mathrm{e}}$, 

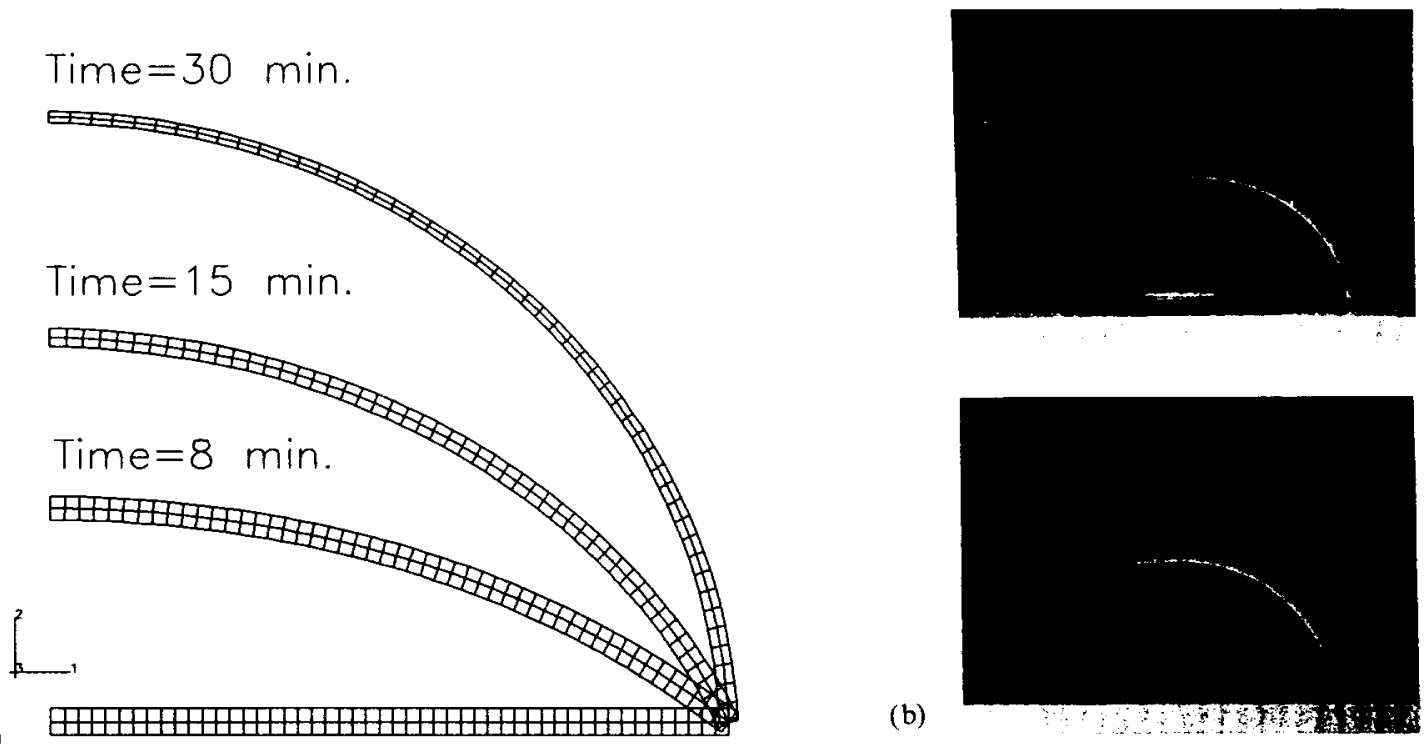

(a)

Fig. 9. (a) Initial and deformed configurations from a finite-element simulation of superplastic free inflation and (b) a specimen and its cut-out.

$\overline{\dot{\varepsilon}}_{\mathrm{p}}=\left(\dot{\varepsilon}_{3}\right)_{\mathrm{p}}$,

and

$\bar{\sigma}_{\mathrm{e}}=\frac{\sqrt{3}}{2}\left(\sigma_{\mathrm{l}}\right)_{\mathrm{e}}$,

$\bar{\sigma}_{\mathrm{p}}=\left(\sigma_{1}\right)_{\mathrm{p}}$,

Assuming no bending and shear effects, equilibrium dictates (Fig. 13)

$\left(\sigma_{1}\right)_{\mathrm{p}} s_{\mathrm{p}}=\left(\sigma_{1}\right)_{\mathrm{e}} s_{\mathrm{e}}$,

that is

$\bar{\sigma}_{\mathrm{p}} s_{\mathrm{p}}=\frac{2}{\sqrt{3}} \bar{\sigma}_{\mathrm{e}} s_{\mathrm{e}}$

If the stress-strain rate relationship is assumed to follow the power law $\bar{\sigma}=K \bar{\varepsilon}^{m}$, then Eq. (35) becomes

$K\left(\dot{\varepsilon}_{3}\right)_{\mathrm{p}}^{m} s_{\mathrm{p}}=K \frac{2}{\sqrt{3}}\left(\frac{2}{\sqrt{3}}\left(\dot{\varepsilon}_{3}\right)_{\mathrm{e}}\right)^{m} s_{\mathrm{e}}$,

or

$\left(\dot{\varepsilon}_{3}\right)_{\mathrm{e}} s_{\mathrm{e}}^{1 / m}=C\left(\dot{\varepsilon}_{3}\right)_{\mathrm{p}} s_{\mathrm{p}}^{1 / m}$

where $C=(\sqrt{3} / 2)^{1+1 / m}$.

Substituting the thickness strain rate, $\dot{\varepsilon}_{3}=\dot{s} / s$, into Eq. (38) we get

$s_{\mathrm{e}}^{(1 / m)-{ }^{1}} \dot{s}_{\mathrm{e}}=C s_{\mathrm{p}}^{(1 / m)-1} \dot{s}_{\mathrm{p}}$,

which can be re-written as

$s_{\mathrm{e}}^{(1 / m)-1} \mathrm{~d} s_{\mathrm{e}}=C s_{\mathrm{p}}^{(1 / m)-1} \mathrm{~d} s_{\mathrm{p}}$

Thus, the thickness relationship between the equator and the pole can be obtained by integrating both sides of the above equation from the initial thickness, $s_{0}$, to the corresponding values at any instant of time $\int_{s_{0}}^{s_{\mathrm{e}}(t)} s_{\mathrm{e}}^{(1 / m)-1} \mathrm{~d} s_{\mathrm{e}}=C \int_{s_{0}}^{s_{\mathrm{p}}(t)} s_{\mathrm{p}}^{(1 / m)-1} \mathrm{~d} s_{\mathrm{p}}$,

to give

$\frac{s_{\mathrm{e}}^{1 / m}(t)-s_{0}^{1 / m}}{\frac{1}{m}}=C \frac{s_{\mathrm{p}}^{1 / m}(t)-s_{0}^{1 / m}}{\frac{1}{m}}$,

or

$s_{\mathrm{e}}(t)=\left[(1-C) s_{0}^{1 / m}+C s_{\mathrm{p}}^{1 / m}(t)\right]^{m}$

Substituting this relationship into Eq. (27), the only unknown, $s_{\mathrm{p}}(t)$, in the equation can be determined from constancy of volume. That is

$2 \pi \rho^{2} \int_{0}^{R} \frac{r\left(s_{\mathrm{p}}+\left[\left((1-C) s_{0}^{1 / m}+C s_{\mathrm{p}}^{1 / m}\right)^{m}-s_{\mathrm{p}}\right]\left(\frac{l}{L}\right)^{2}\right)}{\sqrt{\rho^{2}-r^{2}}} \mathrm{~d} r$
$\quad=\pi R^{2} s_{0}$,

in which $l$ can be related to $r$ as $(l / L)=\sin ^{-1}(r / \rho) / \sin$ $1(R / \rho)$.

If Eq. (44) can be solved to give $s_{\mathrm{p}}(t)$ as a function $h(t)$, then the stress $\left(\bar{\sigma}=p \rho / 2 s_{p}\right)$ and strain rate $\left(\dot{s}_{p} / s_{p}\right)$ at the pole can be obtained from the measured $h(t)$ in the same manner as the uniform-thinning case, to yield the needed material parameters. Unfortunately, a closed-form solution for $s_{\mathrm{p}}$ does not exist. However, as depicted in Appendix $\mathrm{C}$, it is possible to set up $s_{\mathrm{p}}$ as a function of $t$ and $m$ by solving Eq. (44) numerically from the known $h(t)$ and some value of $m$, which usually varies between 0.3 and 0.9 . Once this function is determined, the material parameters, for example, $K$ and $m$ in the power law, can be obtained by solving the following minimization problem

$\min _{(K, m)} \sum_{i=1}^{n}\left(\frac{p \rho}{2 s_{\mathrm{p}}}-K\left(\frac{\dot{s}_{\mathrm{p}}}{s_{\mathrm{p}}}\right)^{m}\right)_{\left.t=t_{i}, h=h_{i}\right)}^{2}$ 


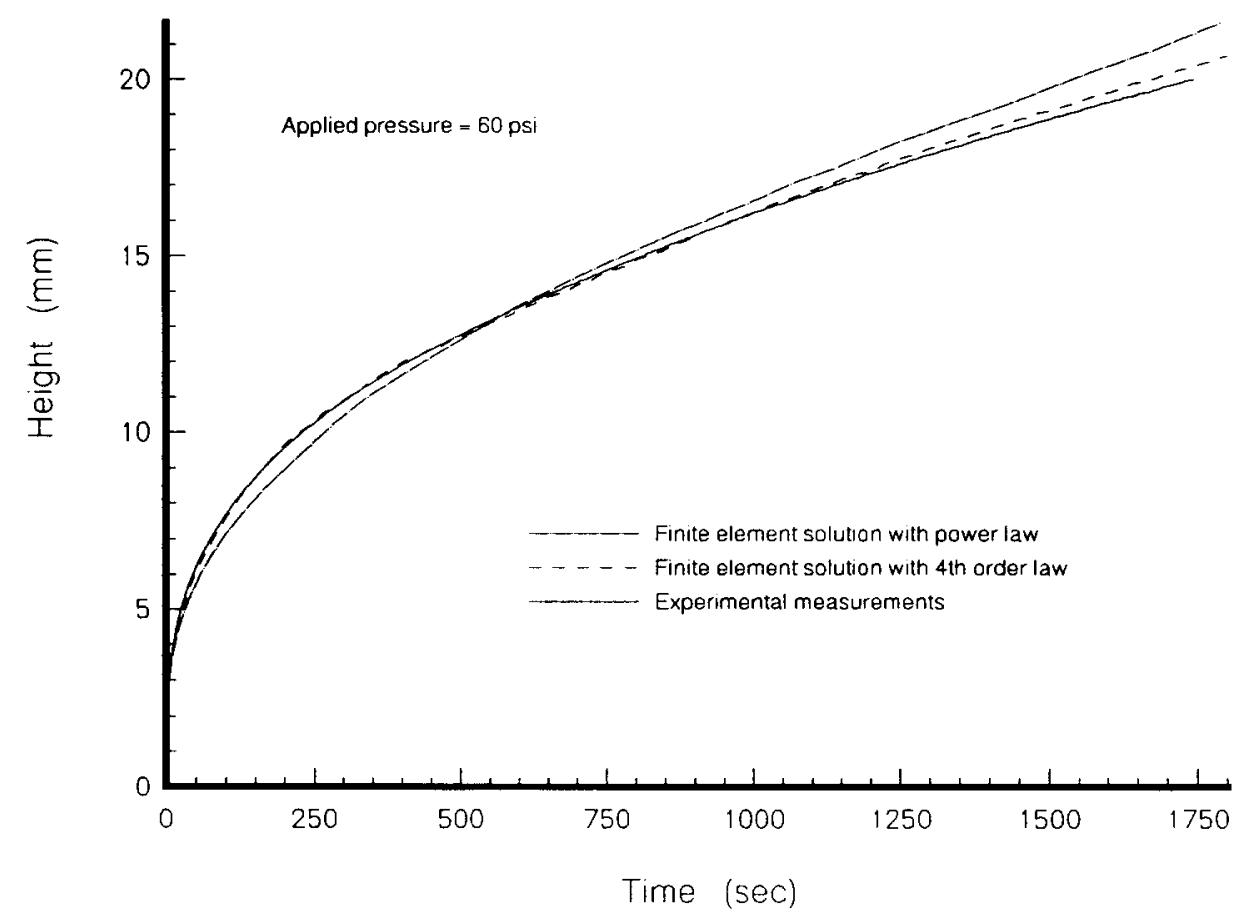

Fig. 10. Comparison of finite-element predictions with experimentally-measured heights of a bulging hemispherical dome.

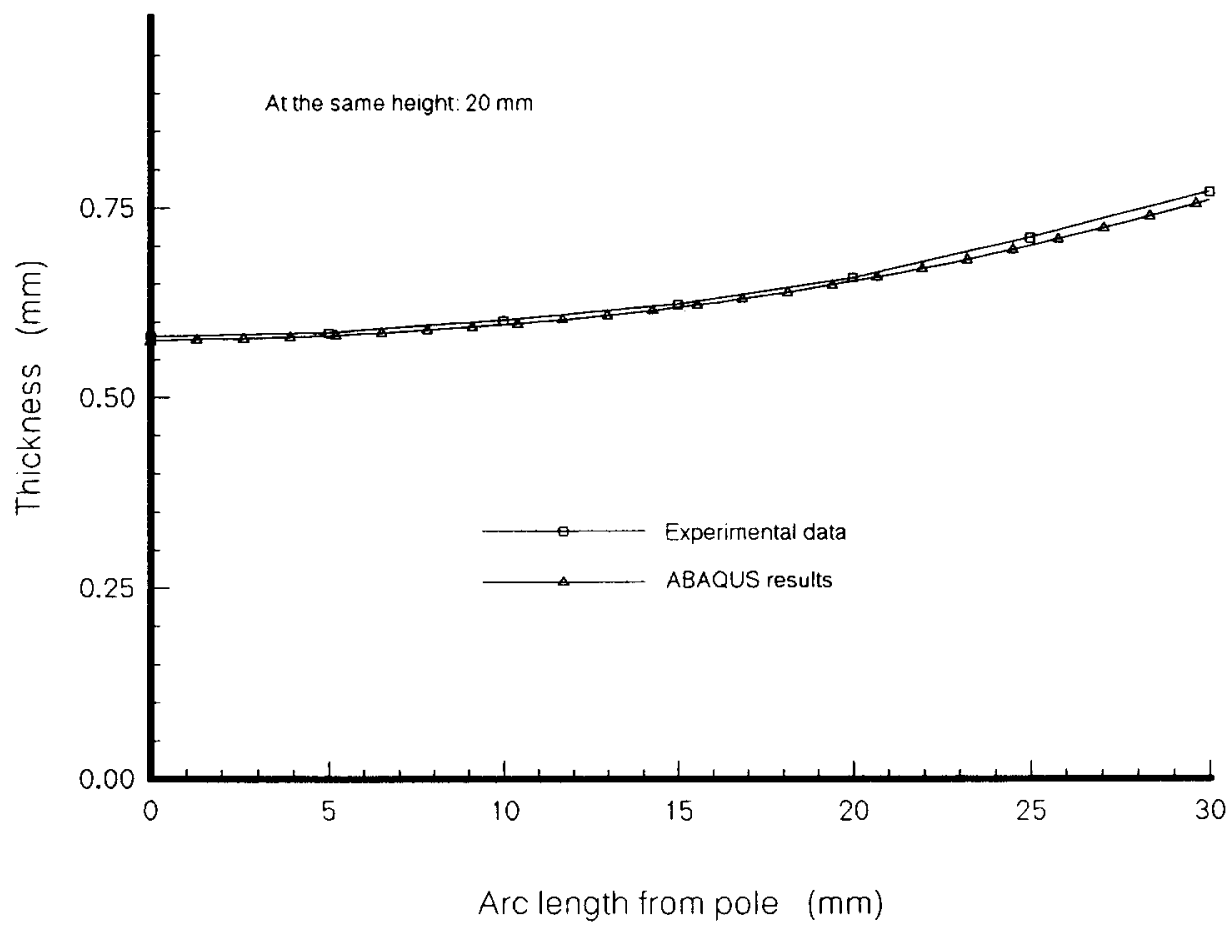

Fig. 11. Comparison of measured and computed thickness-variations along the arc length of the hemisphere.

The summation goes through the measured points $\left\{h\left(t_{i}\right), t_{i}\right\}_{i=1}^{n}$.

The minimization scheme used in Appendix $\mathrm{C}$ is a simple univariate method [20]. The initial guess for the minimization loop is taken from the uniform-thinning solutions. Using the experimental data given in the previous section, $K=1053.021 \mathrm{MPa}$ and $m=0.61293$ are obtained which are not different from the values acquired previously under the assumption of uniform thinning. 


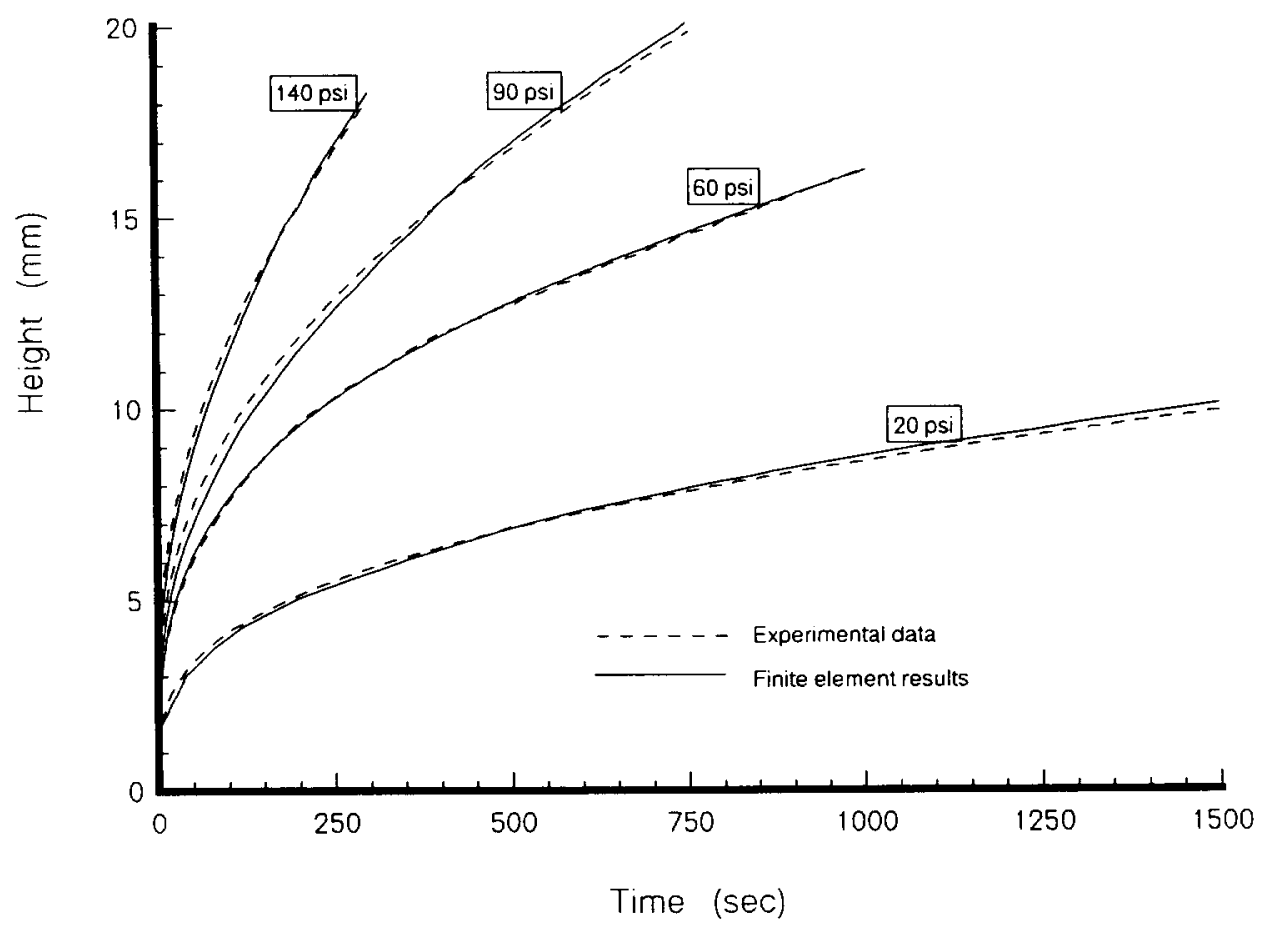

Fig. 12. Finite-element predictions of inflation heights compared with measured data for four different applied pressures.

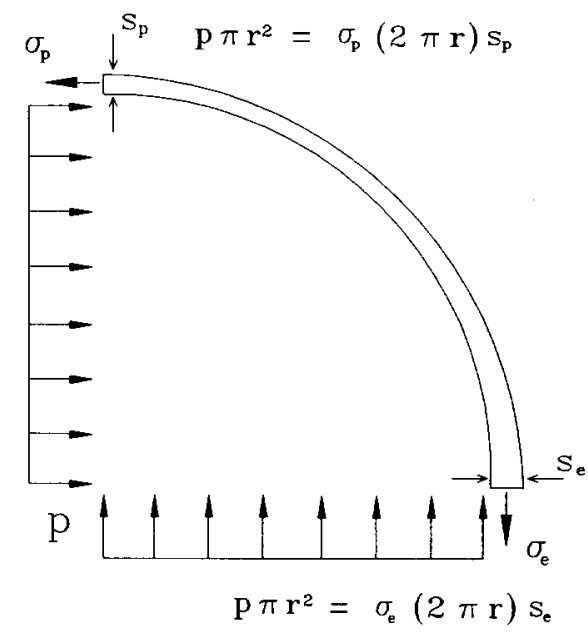

Fig. 13. Force equilibrium between stresses and pressure.

The above results do not mean that taking thickness variation into account is not important. The closeness of the values obtained from the two different programs actually reconfirms the validity of the proposed method. It was mentioned before that the procedure was to tactically adopt only the data when the height of the dome is relatively low, and thus uniformity of the thickness is maintained to some extent.

\section{Conclusions}

Accurate material models and parameters are of vital importance in obtaining reliable finite-element solutions.

A procedure has been proposed for determining parameters of the selected material models from a superplastic blow-forming test. The cost of the experimental devices of the present method is only one-tenth of that of the high-precision tensile-test equipment used conventionally for the same purpose.

Theoretical formulations have been derived to correlate the true stresses and true strain rates from the experimental results. The material models, together with the associated parameters, have been implemented as user's sub-routines for the commercial finite-element program, ABAQUS. The proposed approach has been verified by comparing the numerical solutions obtained from the program with the experimental results.

\section{Acknowledgements}

This research has been sponsored by the National Science Council of the Republic of China under Grant No. NSC-83-0416-E-002-003. 
Appendix A

This appendix contains a Macsyma program for obtaining material parameters $K$ and $m$ of a power law, $\bar{\sigma}=K \bar{\varepsilon}^{m}$, from a superplastic free-inflation test. Derivations of the stress and strain rate are based on the assumption of uniform thinning of the bulging dome.

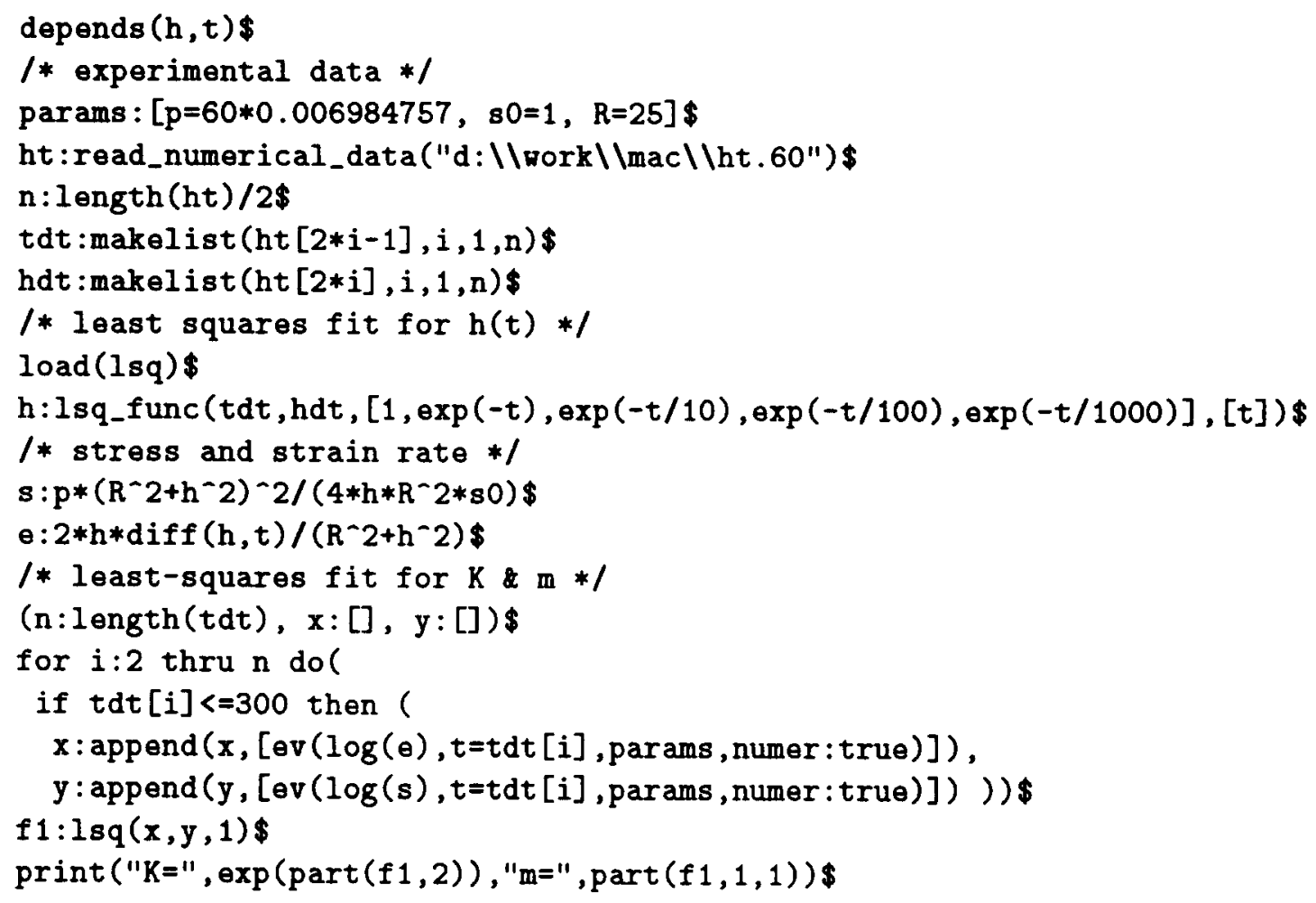

\section{Appendix B}

This appendix contains a Macsyma program for obtaining the strain-rate sensitivity parameter $m=$ $\mathrm{d} \log \bar{\sigma} / \mathrm{d} \log \bar{\varepsilon}$ as a function of strain rate from a superplastic free-inflation test.

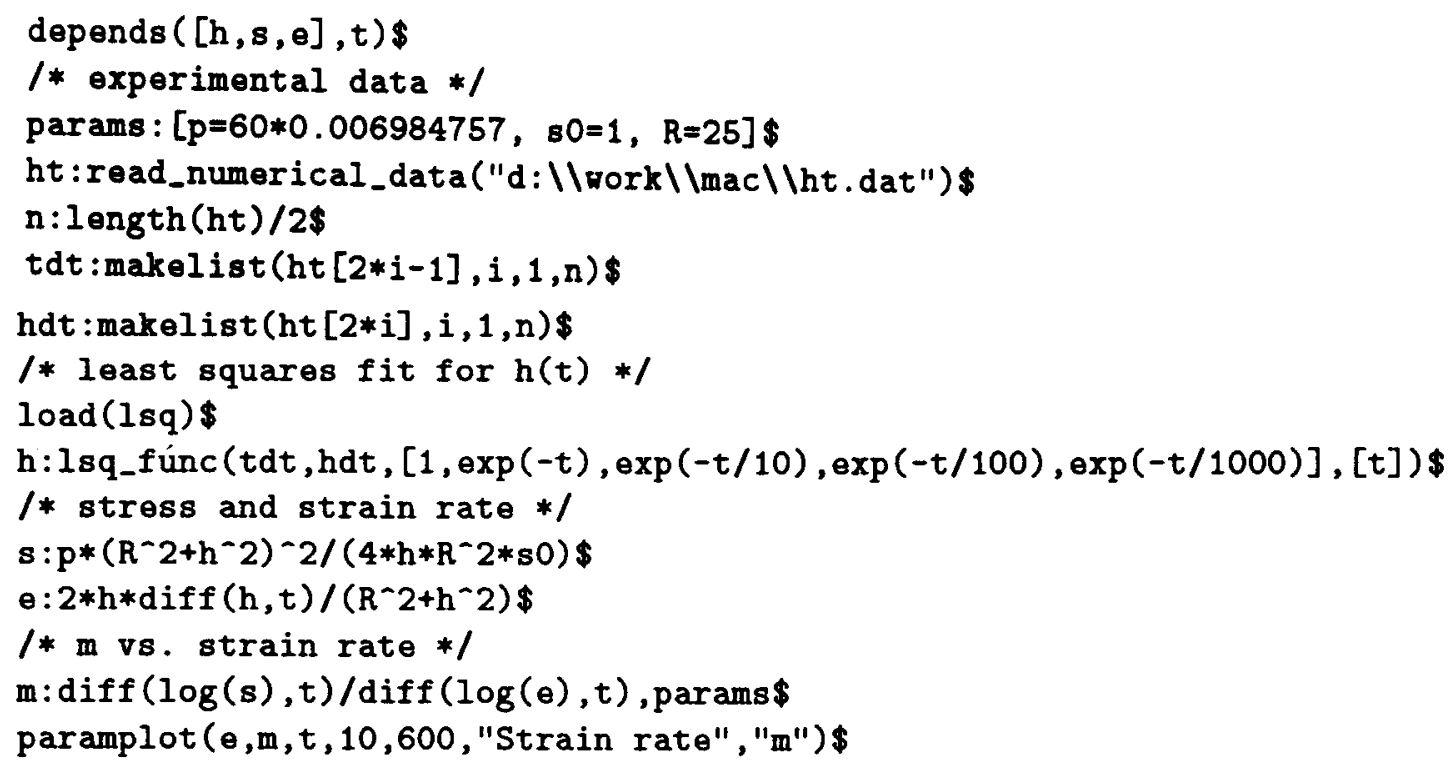




\section{Appendix C}

This appendix contains a Macsyma program for obtaining material parameters $K$ and $m$ of a power law, $\bar{\sigma}=K \overline{\bar{\varepsilon}}^{m}$, from a superplastic free-inflation test. The derivations of stress and strain rate are modified by incorporating a more realistic thickness distribution of the bulging dome.

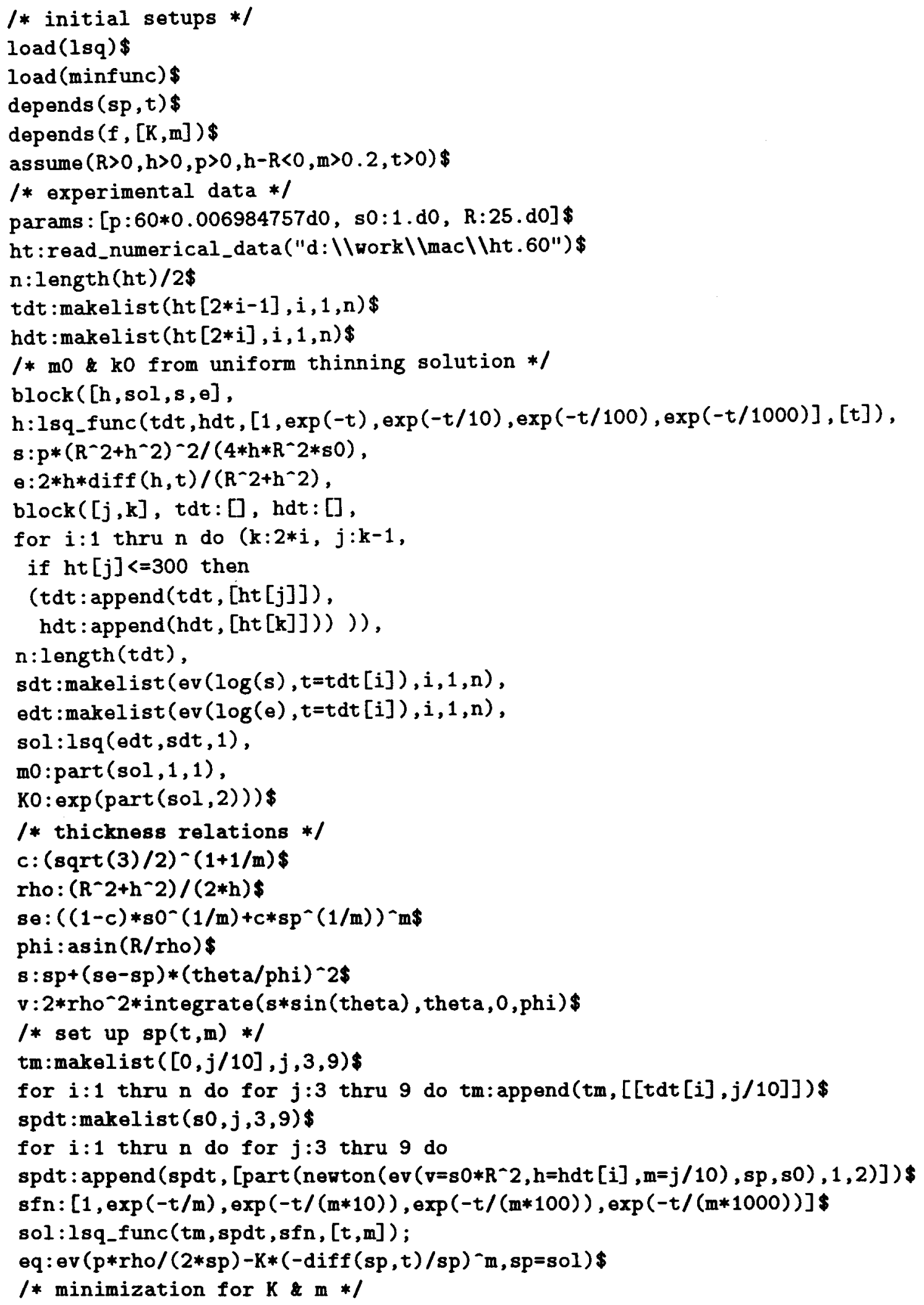




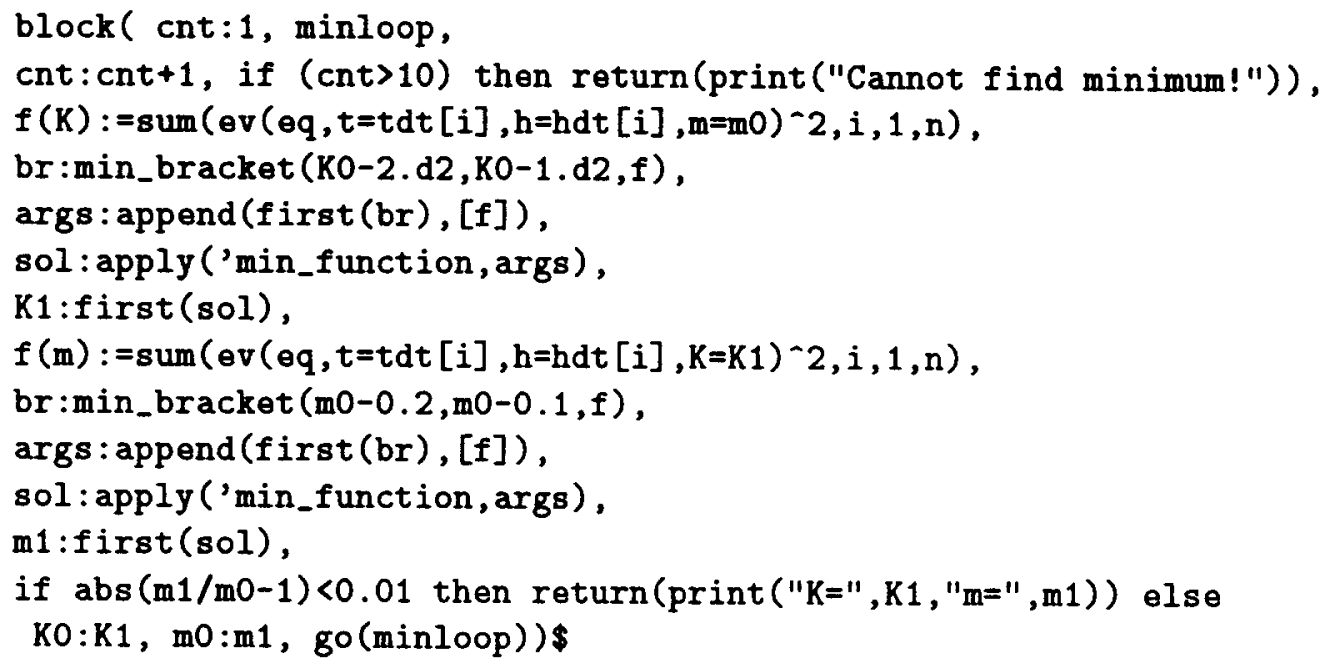

\section{References}

[1] K.A. Padmanabhan and G.J. Davies, Superplasticity, SpringerVerlag, Berlin, 1980, pp. 29-38.

[2] C.H. Hamilton and A.H. Ghosh, Superplastic sheet forming, Metals Handbook, 9th edn., Vol, 14, ASM, Metals Park, 1988, pp. 852-873.

[3] J. Pilling and N. Ridley, Superplasticity, Inst. of Metals, London, 1989 , pp. $48-60$.

[4] O.A. Kaibyshev, Superplasticity of Alloys, Intemetallides, and Ceramics, Springer-Verlag, Berlin, 1992, pp. 4-20.

[5] W.A. Backofen, I.R. Turner and D.H. Avery, Superplasticity in an Al-Zn alloy, Trans. ASM Q., 57 (1964) 980-990.

[6] A.K. Ghosh, in N.E. Paton and C.H. Hamilton (eds.), Superplastic Forming of Structural Alloys, TMS-AIME, Warrendale, 1982, pp. 85-103.

[7] D.L. Holt and W.A. Backofen, Trans. Am. Soc. Met., 59 (1966) 755.

[8] W.B. Morrison, Trans. Am. Soc. Met., 61 (1968) 423.

[9] H. Naziri and R. Pearce, J. Inst. Met., 97 (1969) 326.

10] D. Lee, Strain rate dependent plastic flow behavior of zirconium and its alloys, Metall. Trans., (1970) 1607.
[11] J. Hedworth and M.J. Stowell, J. Mater. Sci., 6 (1971) 1061 1069 .

[12] K. Nuttall, Room-temperature deformation characteristics of the superplastic Zn-Al Eutectoid Alloy, Int. J. Mech. Sci., 13 (1971) 83.

[13] G. Herriot, M. Surey, and B. Baudelet, Superplastic behavior of the industrial Cu7wt.\%P alloy, Scripta Metall, 6 (1972) 657.

[14] M. Suery and B. Baudelet, Flow stress and microstructure in superplastic 60/40 brass, J. Mater. Sci., 8 (1973) 363-369.

[15] A. Nadai, Plasticity, McGraw-Hill, New York, (1931) pp. $77-$ 79.

[16] Macsyma, Mathematics Reference Manual, Macsyma Inc., 1993.

[17] ABAQUS User's Manual, Version 5.3, 1993, p. 5.2.1.

[18] A.K. Ghosh and C.H. Hamilton, Influences of material parameters and microstructure on superplastic forming, Metall. Trans. $A, 13 A$ (1982) $733-743$.

[19] J.H. Cheng, A procedure for designing initial thickness variation for superplastic free inflation, to appear in $\operatorname{lnt} . J$. Mech. Sci., 36 (1994) $981-1000$.

[20] G.N. Vanderplaats, Numerical Optimization Techniques for Engineering Design, McGraw-Hill, New York, 1984. 